\title{
HYDROSTATIC STRESS EFFECT ON THE YIELD BEHAVIOR OF INCONEL 100
}

\author{
Phillip A. Allen and Christopher D. Wilson \\ Department of Mechanical Engineering \\ Tennessee Technological University \\ P.O. Box 5014 \\ Cookeville, TN 38505-0001 \\ 931-372-3102 voice \\ 931-372-6340 fax \\ paa5443@tntech.edu
}

\begin{abstract}
Classical metal plasticity theory assumes that hydrostatic stress has no effect on the yield and postyield behavior of metals. Recent reexaminations of classical theory have revealed a significant effect of hydrostatic stress on the yield behavior of notched geometries. New experiments and nonlinear finite element analyses (FEA) of Inconel 100 (IN 100) equal-arm bend and double-edge notch tension (DENT) test specimens have revealed the effect of internal hydrostatic tensile stresses on yielding. Nonlinear FEA using the von Mises (yielding is independent of hydrostatic stress) and the Drucker-Prager (yielding is linearly dependent on hydrostatic stress) yield functions was performed. In all test cases, the von Mises constitutive model, which is independent of hydrostatic pressure, overestimated the load for a given displacement or strain. Considering the failure displacements or strains, the Drucker-Prager FEM's predicted loads that were $3 \%$ to $5 \%$ lower than the von Mises values. For the failure loads, the Drucker Prager FEM's predicted strains that were $20 \%$ to $35 \%$ greater than the von Mises values. The Drucker-Prager yield function seems to more accurately predict the overall specimen response of geometries with significant internal hydrostatic stress influence.
\end{abstract}




\section{INTRODUCTION}

Since the 1940's, many have considered Bridgman's experiments on the effects of hydrostatic pressure on metals the definitive study. In his study, Bridgman tested smooth (unnotched) tensile bars made from a variety of common metals including aluminum, copper, brass, bronze, and various steels. He conducted tensile tests under the conditions of externally applied hydrostatic pressures up to $3100 \mathrm{MPa}$ (450 ksi) and found that there was no significant effect on the yield point until the higher pressures were reached. His studies revealed that the primary effect of hydrostatic pressure was increased ductility. Bridgman also measured the volume of the material in the gage section and found that this volume did not change, even for very large changes in plastic strain. Since the volume in the gage section did not change, he concluded that metals have incompressible plastic strains. His two observations about metal behavior—no effect of hydrostatic pressure on yielding and incompressibility for plastic strain changes-have become the standard tenets for studies of metal plasticity [1].

Because of the influence of Bridgman's work, plasticity textbooks from the earliest (e.g. Hill [2]) to the most modern (e.g. Lubliner [3]) infer that there is negligible hydrostatic stress effect on the yielding of metals. Even modern finite element programs such as ANSYS [4] and ABAQUS [5] direct the user to assume the same. Engineers often make calculations based on the assumption that the effect of hydrostatic stress is negligible, when in reality the effects of hydrostatic stress can have a significant influence on material yield behavior. It is well documented that large tensile hydrostatic stresses develop in sharply notched or cracked geometries [6-9].

In the 1970's Spitzig and Richmond [10] found that hydrostatic pressure had a significant effect on the stress-strain response of four steels: 4310,4330 , maraging steel and HY80. For 
example, Spitzig reported that the compressive yield strength of 4330 steel increased from 1520 $\mathrm{MPa}$ to $1610 \mathrm{MPa}$ as pressure was increased to $1100 \mathrm{MPa}$ and that the compressive yield strength of the aged maraging steel increased from $1810 \mathrm{MPa}$ to $1890 \mathrm{MPa}$ as pressure was increased to $1100 \mathrm{MPa}$.

Recently, Wilson [11] has conducted experiments and nonlinear finite element analyses of 2024-T351 notched round bars (NRB) to investigate the effect of hydrostatic tensile stresses on yielding. He modeled the loading of a NRB to failure using the finite element method and the traditional, accepted approach combining a pressure-independent yield function with isotropic hardening. His von Mises (yielding is independent of hydrostatic stress) results overestimated experimental load-displacement curves by 10 to $65 \%$ depending on the notch root acuity. Wilson then conducted finite element analyses (FEA) using a yield function that is linearly dependent on hydrostatic stress, the Drucker-Prager yield function. The Drucker-Prager yield function has often been used for soil mechanics calculations but not for metals. The possibility of using the Drucker-Prager yield function for metals was hinted at by Richmond [12] and is discussed later in this paper. Wilson's Drucker-Prager FEA results essentially matched the experimental test data.

The objective of this study is to examine the effect of hydrostatic stress on the yield behavior of Inconel 100 (IN100). The first research task is to compile load-displacement or load-microstrain test data from IN100 test geometries with varying amounts of hydrostatic tensile stress. The second part of the research is to determine the accurate material properties for IN100 for use in nonlinear finite element analyses. The final research task is to model the behavior of the specimens using hydrostatic independent and hydrostatic dependent constitutive 
models in nonlinear finite element analyses and to compare the FEA results with the actual test data.

\section{CLASSICAL METAL PLASTICITY}

Plastic material behavior is a more complex phenomenon than elastic material behavior. In the elastic range, the strains are linearly related to the stresses by Hooke's law, and the strains are uniquely determined by the stresses. In general, plastic strains are not uniquely determined by the stresses. Plastic strains depend on the whole loading history or how the stress state was reached [13]. Therefore, to completely describe material behavior in the plastic range, one must determine the appropriate yield function, hardening rule, and flow rule.

\section{Yield Functions}

In classical metal plasticity theory, a yield function, $f$, is a function of the three principal stresses $\sigma_{1}, \sigma_{2}$, and $\sigma_{3}$ (the characteristic values or eigenvalues of the stress tensor) [13]. The hydrostatic or mean stress is

$$
\sigma_{m}=\frac{1}{3} I_{1}=\frac{1}{3}\left(\sigma_{1}+\sigma_{2}+\sigma_{3}\right)
$$

and the hydrostatic pressure, $p$ is $-\sigma_{m}$. The term $I_{l}$ is the first invariant of the stress tensor. Assuming that yield is independent of mean stress leads to a yield function

$$
f=f\left(J_{2}, J_{3}\right) .
$$

where,

$$
J_{2}=\frac{1}{6}\left[\left(\sigma_{1}-\sigma_{2}\right)^{2}+\left(\sigma_{2}-\sigma_{3}\right)^{2}+\left(\sigma_{3}-\sigma_{1}\right)^{2}\right]
$$

and 


$$
J_{3}=\left(\sigma_{1}-\sigma_{m}\right)\left(\sigma_{2}-\sigma_{m}\right)\left(\sigma_{3}-\sigma_{m}\right)
$$

The terms $J_{2}$ and $J_{3}$ are the second and third invariants of the deviatoric stress tensor. When $f<0$ the material behaves elastically, and when $f=0$ yielding occurs.

The von Mises yield function is often used for classical metal plasticity calculations. This function states that yield is independent of hydrostatic stress and only depends on $J_{2}$ in the form of

$$
f\left(J_{2}\right)=J_{2}-k^{2}
$$

where $k$ is the yield strength in pure shear and is a function of plastic strain for hardening materials. The von Mises or effective stress is defined as

$$
\sigma_{e f f}=\sqrt{3 J}_{2}^{-}=\sqrt{\frac{1}{2}\left[\left(\sigma_{1}-\sigma_{2}\right)^{2}+\left(\sigma_{2}-\sigma_{3}\right)^{2}+\left(\sigma_{3}-\sigma_{1}\right)^{2}\right]} .
$$

Setting $f\left(J_{2}\right)$ equal to zero in Equation (5) leads to

$$
J_{2}=k^{2},
$$

which can be interpreted as the von Mises yield surface in Haigh-Westergaard (principal stress) space. The yield surface for the von Mises yield function is a circular cylinder of radius, $k$, whose axis is defined in the direction of the hydrostatic pressure (Fig. 1). A yield locus is a curve made by intersecting the yield surface with a plane perpendicular to the cylinder axis. For the von Mises yield function, a yield locus taken anywhere along the hydrostatic pressure axis is a circle of radius $k$, thus denonstrating the function's hydrostatic independence. The hydrostatic stress is zero on the plane passing through principal stress space origin. This plane is denoted as the $\pi$ plane and is given by the equation $\sigma_{1}+\sigma_{2}+\sigma_{3}=0$. 


\section{Hardening Rules}

If a material exhibits strain hardening, the yield surface may change shape or location or both as the material deforms plastically. This effect can be approximated for many materials by using isotropic hardening wherein the yield surface expands equally in all directions. Considering the von Mises yield function, the radius of the yield surface increases from $k_{1}$ to $k_{2}$ as the material hardens.

A material that obeys isotropic hardening has the same yield behavior in both tension and compression. This is approximately true for some materials, but it is not an accurate description of material behavior in general. Many materials exhibit a behavior referred to as the Bauschinger effect, wherein the yield strength for tension is different than that for compression. This effect is very important when a reversal of loading (locally or globally) is to be considered.

A kinematic hardening model can describe the behavior of materials with a significant Bauschinger effect. This is accomplished by shifting the axis of the yield surface in principal stress space while maintaining the same radius as the initial yield surface. Since almost no material hardens in a pure isotropic or kinematic fashion, a linear combination of both models is often used to describe materials.

\section{Flow Rules}

Flow rules for plastic behavior are somewhat analogous to Hooke's law for elastic behavior. Hooke's law defines the relationship between stress and elastic strains, while flow rules define the relationship between stresses and plastic strain increments. A general form of a flow rule relating stresses to plastic strain increments is given by 


$$
d \varepsilon_{i j}^{p l}=\frac{\partial g}{\partial \sigma_{i j}} d \phi
$$

where $d \varepsilon_{i j}^{p l}$ are the plastic strain increments, $g$ is the plastic potential function, and $d \phi$ is a positive constant [13]. Associated flow occurs when $g=f$, where $f$ is the yield function.

For an incompressible material, the sum of the plastic strain increments (or plastic dilatation rate) must be zero. This can be written in terms of the principal strain increments $d \varepsilon_{l}$. $d \varepsilon_{2}$, and $d \varepsilon_{3}$ as

$$
d \varepsilon_{i i}^{p l}=d \varepsilon_{1}^{p l}+d \varepsilon_{2}^{p l}+d \varepsilon_{3}^{p l}=0
$$

where $d \varepsilon_{1}^{p l}$ is the plastic pottion of $d \varepsilon_{1}$. Equation (9) is written in associated form as

$$
d \varepsilon_{i j}^{p l}=\frac{\partial f}{\partial \sigma_{i j}} d \phi
$$

Drucker and Prager [14] showed that $d \varepsilon_{i i}^{p l}$ can be summed from Equation (10) to obtain

$$
d \varepsilon_{i i}^{p l}=3 d \phi \frac{\partial f}{\partial I_{1}}
$$

Since the hydrostatic stress is $1 / 3 I_{1}, d \varepsilon_{i i}^{p l}$ must equal zero if the yield function does not depend on hydrostatic stress.

\section{PRESSURE-DEPENDENT PLASTICITY}

Bridgman continued to study the effects of external hydrostatic pressure for many years, and, in 1952, he wrote a comprehensive summary of his work in his book "Studies in Large Plastic Flow and Fracture with Special Emphasis on the Effects of Hydrostatic Pressure [15]." In this book, he reexamined his earlier results and made observations that many plasticity books failed to notice. On p. 64 of his book, Bridgman writes: 
"By the time the last series of measurements was being made under the arsenal contract, however, skill in making the measurements had so increased, and probably also the homogeneity of the material of the specimens had also increased because of care in preparation, that it was possible to establish a definite effect of pressure on the strain hardening curve [15]."

For example, Bridgman's tests found that the flow stress for tempered pearlite at a strain of 2.75 increased from $1758 \mathrm{MPa}(255 \mathrm{ksi})$ at atmospheric pressure to $2171 \mathrm{MPa}(315 \mathrm{ksi})$ when pressurized to approximately $2482 \mathrm{MPa}$ (360 ksi). Therefore, Bridgman clearly demonstrated in his later work a definite external hydrostatic pressure effect on yielding. Unfortunately, he failed to consider the effect of internally generated hydrostatic stresses.

In the 1970's Richmond, Spitzig, and Sober $[10,12,16]$ also conducted experiments that challenged the basic tenets of classic metal plasticity. They studied the effects of hydrostatic pressure on yield strength for four steels: 4310, 4330, maraging steel, and HY80. They conducted compression and tension tests on smooth specimens in a Harwood hydrostaticpressure unit at pressures up to $1100 \mathrm{MPa}(160 \mathrm{ksi})$.

Richmond found that the yield strength was a linear function of hydrostatic pressure as shown in Fig. 2. Richmond proposed that for high-strength steels the yielding process is described by the yield function

$$
f\left(I_{1}, J_{2}\right)=\sqrt{3 J_{2}}+a I_{1}-d
$$

where $d$ is the modified yield strength in absence of mean stress and $a$ is a material constant related to the theoretical cohesive strength of the material, $\sigma_{c}$. Equation (12) is identical to the yield function originally proposed by Drucker and Prager [14] to solve soil mechanics problems. The material constant $a$ is determined graphically as the slope of the graph of $\sigma_{\text {eff }}$ versus $I_{1}$, as illustrated in Figure 3. The value of $I_{1}$ for $\sigma_{e f f}=0$ is equal to the theoretical cohesive strength of the material, and the value of $I_{1}=\sigma_{\text {uniaxial }}$ corresponds to the yield strength for a tensile test. 
Also, the value of $I_{1}=0$ leads to $\sigma_{e f f}=d$. Values of $a$ and $d$ reported by Richmond [10] and Chen [17] are listed in Table 1.

Another interesting result that emerged from Richmond's tests was a strong correlation between the coefficients $a$ and $d$. He found that the ratio of $a / d$ was nearly constant for all of the steels as listed in Table 1. Richmond also suggested that the ratio ald is a property of the bulk iron lattice similar to the elastic constants $E$ and v.

Richmond also conducted pressurized compression and tension tests on two polymerscrystalline polyethylene and amorphous polycarbonate. These tests were performed to see if the plasticity theories developed for metals were compatible with other materials. He found that hydrostatic pressure had a significant effect on the stress-strain response of the polymers and that the effective stress was a linear function of hydrostatic pressure. In other words, Richmond established that the polymt:rs' plastic response could be described by the same plasticity theories that he developed for metals. Therefore, the fact that soils, metals, and polymers are all affected in a similar manner by hydrostatic pressure is a unifying concept.

The Drucker-Prager yield surface is a right-circular cone in principal stress space as shown in Fig. 1. The axis of the cone is the hydrostatic pressure axis, and the apex of the cone is located at a hydrostatic stress equal to the cohesive strength. The yield surface for an actual material probably does not come to a sharp apex as the linear Drucker-Prager model predicts. The sharp point of the cone could cause numerical difficulty in calculating derivatives for flow calculations, and, therefore, the finite element code ABAQUS provides hyperbolic and exponential Drucker-Prager constitutive models that round off the end of the cone [5]. For small amounts of hydrostatic stress, the cylinder of the von Mises yield criterion can approximate the cone. As the hydrostatic stress increases, the deviation from the cylinder can be considerable, 
and the Drucker-Prager yield surface is preferable (Fig. 1). Because of its hydrostatic dependency, the Drucker-Prager yield criterion should result in more accurate modeling of geometries that have a high hydrostatic stress influence such as cracks and notches.

\section{RESEARCH PROGRAM}

Accurate load-displacement or load-microstrain test data was obtained from Pratt \& Whitney [18] and NASA [19] for two IN100 test geometries: an equal-arm bend specimen (Fig. 4) and a double-edge notch tension (DENT) specimen (Fig. 5). Several nonlinear finite element analyses were then conducted in order to assess how well the von Mises and Drucker-Prager failure FEM's could match the IN100 specimen test data. The FEM's meshes were created using the modeling program Patran [20]. The Patran meshes were translated into an ABAQUS [5] input file, and the job control commands and material properties were added using a text editor. All of the finite elements models were loaded in displacement control with full integration and large strain effects activated. Details of the FEM's are given by Allen [21].

\section{Equal-Arm Bend Specimen}

The first specimen modeled was an IN100 equal-arm bend specimen (Fig. 4). This specimen was used by Pratt \& Whitney [18] in low cycle fatigue testing to simulate the geometry and loading condition of a highly stresses area in the Space Shuttle main rocket engine fuel turbopump housing. The equal-arm bend FEM was created using Q4 plane strain elements (type CPE4 in ABAQUS) and one symmetry plane resulting in a total of 1339 elements. The symmetry plane and boundary conditions are illustrated in Fig. 6. Plane strain elements were used because the thickness to width ratio in the fillet region was approximately 5 to 1 . The 
loading was applied to the finite element model by filling the hole for the loading-pin with elements and applying a displacement to the node in the center of the loading-pin hole. An illustration of the finite element model is given in Fig. 7. The mesh in the fillet region in Fig. 7 is difficult to see and, therefore, a closer view of the mesh in the fillet region is shown in Fig. 8.

\section{Double-Edge Notch Tension Specimen}

The next geometry analyzed was an IN100 double-edge notch tension (DENT) specimen tested by NASA's Marshall Space Flight Center (Fig. 5). Both two-dimensional (2-D) and threedimensional (3-D) FEM's were created since the thickness to width ratio was approximately unity in the notch region. Three 2-D DENT models were created by utilizing a coarse, medium, and fine mesh in the notch region. The 2-D models were created using Q4 plane stress elements (type CPS4 in ABAQUS). Only the gage section of the specimen was modeled by utilizing two planes of symmetry as illustrated in Fig. 9. The coarse, medium and fine meshes had approximately 150,300 , and 630 elements in the notch region, respectively. A convergence study was performed comparing the performance of the three mesh densities, and the medium mesh was chosen as a good balance between computational speed and accuracy. An illustration of the medium density mesh 2-D FEM is given in Fig. 10.

The 3-D DENT finite element model was created using $4740 \mathrm{H} 8$ elements (type C3D8 in ABAQUS). Three symmetry planes were utilized in creating this FEM by dividing the specimen along the longitudinal axis, along the transverse axis, and through one-half of the thickness. The 3-D DENT mesh was created by extruding the 2-D medium density mesh with 10 elements through the thickness. 


\section{Required Material Properties}

Several material properties are required as input for the von Mises and Drucker-Prager constitutive models in ABAQUS. Both constitutive models require the elastic material properties of Young's modulus, $E$, and Poisson's ratio, $v$. The von Mises model with hardening also necessitates a table of true stress, $\sigma$, versus plastic strain, $\varepsilon_{p l}$. The elastic material properties and the table of $\sigma$ versus $\varepsilon_{p l}$ for Inconel 100 (IN100) were gathered from Pratt and Whitney tensile test data [22]. The true stress versus true strain plots for IN100 at room temperature is given in Fig. 11. It is notable that IN100 has an upper and lower yield point.

The plastic material properties for the Drucker-Prager constitutive model require careful explanation and, therefore, will be discussed in some detail. The linear Drucker-Prager yield function is written in ABAQUS as

$$
f=t-p \tan \beta-d,
$$

where

$$
t=\frac{1}{2} \sqrt{3 J_{2}}\left[1+\frac{1}{K}-\left(1-\frac{1}{K}\right)\left(\frac{r}{\sqrt{3 J_{2}}}\right)^{3}\right]
$$

$\beta$ is the slope of the linear yield surface in the $p-t$ stress plane, $p$ is the hydrostatic pressure, $d$ is the effective cohesion of the material, and $K$ is the ratio of the yield stress in triaxial tension to the yield stress in triaxial compression. The variables used by the linear Drucker-Prager yield function are shown graphically in Fig. 12.

The flow potential, $g$, for the linear Drucker-Prager model is defined as

$$
g=t-p \tan \psi,
$$


where $\psi$ is the dilation angle in the $p$ - $t$ plane (Fig. 12). The dilation angle controls the movement of an arbitrary point on the yield surface during the hardening process. Setting $\psi=\beta$ results in associated flow. Therefore, the original Drucker-Prager model is available by setting $\psi=\beta$ and $K=1$ [5]. Associated flow was used in this research as a first-order approximation.

In order to conveniently compare ABAQUS Drucker-Prager material property variables with those used by Richmond, et al. in their material testing, one must correlate the variables in Equations (12) and (13). Recalling that $p=-1 / 3 I_{1}$ and $t=\sqrt{3 J_{2}}$ for $K=1$, Equation (13) can be written as

$$
f=\sqrt{3 J_{2}}+\frac{1}{3} I_{1} \tan \beta-d
$$

Comparing Equations (12) and (16) leads to the conclusion that

$$
a I_{1}=\frac{1}{3} I_{1} \tan \beta
$$

Solving for $\beta$ yields

$$
\beta=\tan ^{-1}(3 a)
$$

thus demonstrating how the material constants of the original Drucker-Prager theory and the material constants used in the ABAQUS linear Drucker-Prager constitutive model are be related.

The linear Drucker-Prager constitutive model in ABAQUS requires, in addition to the elastic constants, values for $\beta, \psi, K$, and a table of modified effective stress, $\tilde{\sigma}_{\text {eff }}$, versus $\varepsilon_{p l}$. The modified effective stress is defined as

$$
\tilde{\sigma}_{e f f}=\sigma_{e f f}(1-a)
$$

The factor (1-a) is a small correction to account for the presence of hydrostatic stress in a smooth tensile test data. Since the values of $a$ for a metal are typically very small, the correction 
to the $\sigma_{\text {eff }}$ versus $\varepsilon_{p l}$ curve is correspondingly small. For example, using Richmond's reported value of $a$ for 4310 steel (Table 1) results in a 2.5 percent lower $\sigma_{e f f}$ versus $\varepsilon_{p l}$ curve.

The challenge comes in calculating a value for $a$. It was reported earlier that the ratio of a/d in Richmond's tests [10] was nearly constant for the high strength steels, and, therefore, ald is possibly a material constant similar to Young's modulus or Poisson's ratio. Since Young's modulus for IN100 is similar to that of high strength steels, it was assumed that $a$ for IN100 is approximately equal to the values of $a$ reported by Richmond (Table 1). Estimating a value for $a$ for other metals can be more difficult though because no extensive test data of $\sigma_{\text {eff }}$ versus $I_{l}$ is readily available. The value for $a$ can be estimated for any metal by conducting uniaxial tension and uniaxial compression tests and plotting the yield results in $\sigma_{\text {eff }}$ versus $I_{1}$ space. The slope $a$ of the line connecting the compressive yield, $\sigma_{y s c}$, and the tensile yield, $\sigma_{y s}$, in Fig. 3 is

$$
a=\frac{\sigma_{y s c}-\sigma_{y s}}{\sigma_{y s c}+\sigma_{y s}}
$$

Dieter [23] suggests that for many metals the material cohesive strength, $\sigma_{c}$, can be estimated as $E / 15$ to $E / 4$ with a typical value of $E / 5.5$. Therefore, $a$ can also be estimated as initial yield strength divided by an approximate cohesive strength.

\section{RESULTS AND CONCLUSIONS}

\section{IN100 Material Properties}

Poisson's ratio for IN100 was assumed to be 0.30 . The Lüder effect from the upper and lower yield strength was neglected in constructing the true stress versus plastic strain curve, and, therefore, the lower yield stress was used for $\sigma_{y s}$. To use the original form of the linear Drucker- 
Prager constitutive model, $K$ was set equal to one, and $\beta$ and $\psi$ were equated. Richmond's test results (Table 1) for high strength steels was used to estimate a value for $a$ of 0.022 . Using Equation (18), $\beta$ was calculated to be approximately $3.8^{\circ}$. The values of $\tilde{\sigma}_{\text {eff }}$ were calculated for increments of $\varepsilon_{p l}$ using Equation (19).

Pratt and Whitney [22] tensile test data for IN100 has widely scattered values for $E$ ranging from 172.4 to $241.3 \mathrm{GPa}\left(25 \times 10^{6}\right.$ to $\left.35 \times 10^{6} \mathrm{psi}\right)$. Young's modulus was assumed to be $227.5 \mathrm{GPa}\left(33.0 \times 10^{6} \mathrm{psi}\right)$ for the DENT models to match the test data in the linear range and 179.3 $\mathrm{GPa}\left(26.0 \times 10^{6} \mathrm{psi}\right)$ for the equal-arm bend model. The reason for the change in $E$ is a matter of debate, but it is possible that the measured value of $E$ for IN100 changes with loading type [24]. This is supported by the fact that in addition to normal stresses, the equal-arm bend specimen was under the influence of large bending stresses.

\section{Equal-Arm Bend Comparisons}

Pratt \& Whitney tt:st data [18] was provided for a five-cycle proof test, but only the initial loading portion of the first cycle was considered in this research. Load versus microstrain FEM curves for the equal-arm bend specimen are plotted alongside the Pratt \& Whitney test data in Fig. 13. The FEM strain data is the maximum strain at the integration points of the element at the fillet root. The von Mises model overestimates the load for a given value of strain in the postyield region. Considering the strain at load reversal, the Drucker-Prager FEM predicts loads that are about 5\% lower than the von Mises values. For the maximum load, the Drucker Prager FEM predicts strains that are approximately $20 \%$ greater than the von Mises values, and, therefore, does a much better job of matching the overall specimen response. 


\section{Double-Edge Notch Tension Comparisons}

Load-displacement data for the medium mesh 2-D and 3-D DENT finite element models are plotted alongside NASA test data [19] in Fig. 14. The test data crosses the FEM curves at a strain of approximately 0.0003 . For all of the FEM's, the von Mises yield criterion overestimates the load for a given value of displacement in the postyield region. Considering the failure displacement, the Drucker-Prager FEM's predict loads that are about 3\% lower than the von Mises values. For the failure load, the Drucker Prager FEM's predict strains that are about 35\% greater than the von Mises values. The 2-D plane stress model gives load values that are about $2 \%$ lower than those given by the 3-D model, illustrating the lower stiffness of the 2-D model. A plane strain model was also tried, but the results of this FEM greatly overestimated the test data load for a given value of displacement in the postyield region. Overall, the DENT finite element models did not predict test specimen behavior as well as the equal-arm bend FEM.

\section{Conclusions}

The following are the conclusions reached from this study of hydrostatic stress effects in yield behavior of IN100.

1. Internally generated hydrostatic pressure does have a significant effect on the yield and postyield behavior of IN100.

2. In all test cases, the von Mises constitutive model, which is independent of hydrostatic pressure, overestimated the load for a given displacement or strain.

3. The Drucker-Prager constitutive model, which is a function of hydrostatic stress, produced results that better matched the test data. Considering the failure displacements or strains, the Drucker-Prager FEM's predicted loads that were 3\% to 5\% lower than the 
von Mises values. For the failure loads, the Drucker-Prager FEM's predicted strains that were $20 \%$ to $35 \%$ greater than the von Mises values.

4. This level of increase in accuracy may be of little interest to the designer using large factors of safety, but, to the high performance designer, a $10 \%$ increase in accuracy is significant.

5. The Drucker-Prager yield function seems to more accurately predict the overall specimen response of geometries with significant hydrostatic influence.

\section{ACKNOWLEDGEMENTS}

We would like to thank Greg Swanson and numerous other members of the Strength Analysis Group at Marshall Space Flight Center for their help and guidance in conducting this study. Funding for this research was provided by the National Aeronautics and Space Administration (NASA) Graduate Student Researchers Program (GSRP). 


\section{NOMENCLATURE}

\begin{tabular}{|c|c|}
\hline Symbol & Description \\
\hline$a$ & Slope of Effective Stress Versus the First Stress Invariant \\
\hline$d$ & Modified Yield Strength \\
\hline$f$ & Yield Function \\
\hline$g$ & Plastic Potential Function \\
\hline$k$ & Yield Strength in Pure Shear \\
\hline$p$ & Hydrostatic Pressure \\
\hline$t$ & Variable in Drucker-Prager Yield Function in ABAQUS \\
\hline$E$ & Young's Modulus \\
\hline$I_{1}$ & First Stress Invariant \\
\hline$J_{2}, J_{3}$ & Second and Third Deviatoric Stress Invariants \\
\hline$P$ & Load \\
\hline$\beta$ & Angle of the Slope of the Yield Surface in the $p$ - $t$ stress plane \\
\hline$d \phi$ & Positive Constant in General Flow Rule \\
\hline$\varepsilon$ & True Strain \\
\hline$\varepsilon_{1}, \varepsilon_{2}, \varepsilon_{3}$ & Principal Strains \\
\hline$\varepsilon_{p l}$ & Plastic Strain \\
\hline$\varepsilon_{e q}^{p l}$ & Equivalent Plastic Strain \\
\hline$\mu \varepsilon$ & Microstrain \\
\hline$v$ & Poisson's Ratio \\
\hline$\sigma$ & True Stress \\
\hline$\sigma_{1}, \sigma_{2}, \sigma_{3}$ & Principal Stresses \\
\hline$\sigma_{c}$ & Theoretical Cohesive Strength \\
\hline$\sigma_{e f f}$ & Effective Stress \\
\hline$\tilde{\sigma}_{e f f}$ & Modified Effective Stress \\
\hline$\sigma_{m}$ & Mean Stress \\
\hline$\sigma_{x x}, \sigma_{y y,} \sigma_{z z}$ & Normal Stresses \\
\hline$\sigma_{y s}$ & Yield Strength \\
\hline$\sigma_{y s c}$ & Compressive Yield Strength \\
\hline$\tau_{x y,}, \tau_{x z}, \tau_{y z}$ & Shear Stresses \\
\hline$\psi$ & Dilation Angle \\
\hline DENT & Double-Edge Notch Tension \\
\hline FEA & Finite Element Analysis \\
\hline FEM & Finite Element Model \\
\hline NASA & National Aeronautics and Space Administration \\
\hline
\end{tabular}




\section{REFERENCES}

[1] Bridgman, P.W., 1947, "The Effect of Hydrostatic Pressure on the Fracture of Brittle Substances," Journal of Applied Physics, Vol. 18, p. 246.

[2] Hill, R., 1950, "The Mathematical Theory of Plasticity," Clarendon Press, Oxford.

[3] Lubliner, J., 1990, "Plasticity Theory," Macmillan, New York.

[4] Kohnke, Peter, ed., 1994, "ANSYS User's Manual for Revision 5.0," Volume IV: Theory.

[5] ABAQUS Theory Manual, Version 5.5, 1995, Hibbit, Karlsson, and Sorensen, Inc.

[6] Neuber, Heinz, 1958, "Theory of Notch Stresses: Principles for Exact Calculation of Strength with Reference to Structural Form and Material," U.S. Atomic Energy Commission, ACE-tr4547, Translated from publication of Springer-Verlag, Berlin, Gottingen, Heidelberg.

[7] Hertzberg, Richard W.. 1996, "Deformation and Fracture Mechanics of Engineering Materials," John Wiley \& Sons, Inc., New York.

[8] Rice, J.R., and D.M. Tracey, 1969, "On the Ductile Enlargement of Voids in Triaxial Stress

Fields," Journal of the Mechanics and Physics of Solids, Volume 17, pp. 201-217.

[9] Gurson, A.L., 1977, "Continuum Theory of Ductile Rupture by Void Nucleation and Growth: Part 1 - Yield Criteria and Flow Rules for Porous Ductile Media," Journal of Engineering Materials and Technology, Volume 99, pp 2-15.

[10] Richmond, O., and W.A. Spitzig, 1980, "Pressure Dependence and Dilatancy of Plastic Flow," International Union of Theoretical and Applied Mechanics Conference Proceedings, pp. 377-386.

[11] Wilson, Christopher D., 2002, "A Critical Reexamination of Classical Metal Plasticity," The Journal of Applied Mechanics, Volume 69, pp. 63-68.

[12] Spitzig, W.A., R.J. Sober, and O. Richmond, 1975, "Pressure Dependence of Yielding and Associated Volume Expansion in Tempered Martensite," ACTA Metallurgica, Volume 23, pp. 885-893.

[13] Mendelson, Alexander, 1983, 'Plasticity: Theory and Application," Krieger Publishing, Florida, originally published by Macmillan, 1968.

[14] Drucker, D.C., and W. Prager, 1952, "Soil Mechanics and Plastic Analysis for Limit Design," Quarterly of Applied Mathematics, Volume 10, pp. 157-165.

[15] Bridgman, P.W., 1952, "Studies in Large Plastic Flow and Fracture with Special Emphasis on the Effects of Hydrostat ic Pressure," McGraw-Hill, New York.

[16] Spitzig, W.A., R.J. Sober, and O. Richmond, 1976, "The Effect of Hydrostatic Pressure on the Deformation Behavior of Maraging and HY-80 Steels and its Implications for Plasticity Theory," Metallurgical Transactions A, Volume 7A, pp. 377-386.

[17] Chen, W.F., and X.L. Liu, 1990, "Limit Analysis in Soil Mechanics," Elsiver, New York. [18] Pratt \& Whitney, Nov. 16, 1999, Report Number MME 42030.

[19] McGill, Preston, August 1999, DENT Test Data, ED33 Metallic Materials and Processes Group, NASA George C. Marshall Space Flight Center.

[20] MSC Software Corporation, 2000, "Patran Users Manual", Version 9.0.

[21] Allen, Phillip A., 2000), "Hydrostatic Stress Effects in Metal Plasticity," Masters Thesis, Tennessee Technology University.

[22] Pratt and Whitney, 1991, "Alternate Turbopump Development (ATD) Materials Manual." [23] Dieter, G.E., 1976, 'Mechanical Metallurgy," McGraw-Hill, New York. 
[24] American Society for Testing and Materials, 2000, 'E855-90, Standard Test Methods for Bend Testing of Metallic Flat Materials for Spring Applications Involving Static Loading," Annual Book of ASTM Standards, ASTM, PA. 


\section{LIST OF TABLES AND FIGURES}

Table 1 Summary of Experimental Results for Constants in Equation (12) [[10],[17]]

Figure 1 Drucker-Prager and von Mises Yield Surfaces in Principal Stress Space

Figure 2 Dependence of Yielding on Mean Stress in 4330 Steel [10]

Figure 3 Schematic of $\sigma_{e f f}$ versus $I_{1}$

Figure 4 Engineering Drawing of the Equal Arm Bend Specimen (Dimensions in mm)

Figure 5 Engineering Drawing of the DENT Specimen (Dimensions in $\mathrm{mm}$ )

Figure 6 Schematic of the Bqual-Arm Bend FEM Utilizing One Symmetry Plane

Figure 7 Equal Arm Bend Finite Element Model Utilizing One Plane of Symmetry

Figure 8 Mesh in the Fillet Region of the Equal Arm Bend Specimen

Figure 9 Schematic of the 2-D DENT FEM Utilizing Two Symmetry Planes

Figure 10 Illustration of the 2-D DENT FEM

Figure 11 True Stress versus True Strain Curve for IN100 at Room Temperature [22]

Figure 12 Linear Drucker-Prager Model: Yield Surface and Flow Direction in the $p-t$ Plane (Adapted from [5])

Figure 13 Load-Microstrain Results for the Equal-Arm Bend Specimen

Figure 14 Load-Displacement Results for the DENT Specimen 
Table 1 Summary of Experimental Results for Constants in Equation (12) [[10],[17]]

\begin{tabular}{lccc}
\hline \multicolumn{1}{c}{ Material Name } & $a$ & $d, \mathrm{MPa}$ & $a / d, \mathrm{MPa}^{-1}$ \\
\hline HY80 Steel & 0.008 & 606 & $13 \times 10^{-6}$ \\
Unaged Maraging Steel & 0.017 & 1005 & $17 \times 10^{-6}$ \\
4310 Steel & 0.025 & 1066 & $23 \times 10^{-6}$ \\
4330 Steel & 0.025 & 1480 & $17 \times 10^{-6}$ \\
Aged Maraging Steel & 0.037 & 1833 & $20 \times 10^{-6}$ \\
Polyethylene & 0.022 & 13 & $17 \times 10^{-4}$ \\
Polycarbonate & 0.011 & 36 & $31 \times 10^{-5}$ \\
Clay & 0.118 & $6.7 \times 10^{-2}$ & 1.76 \\
\hline
\end{tabular}




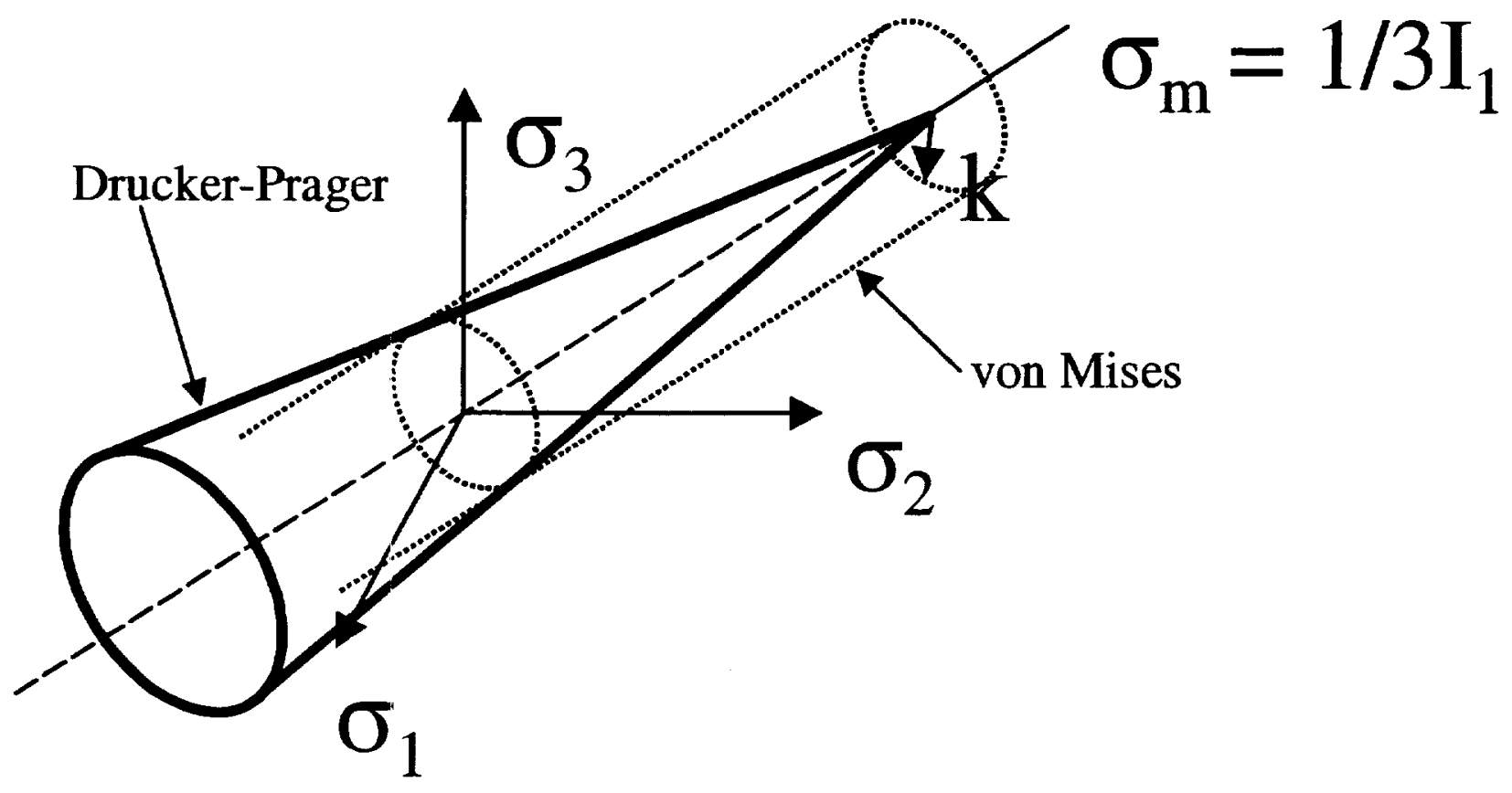

Figure 1 Drucker-Prager and von Mises Yield Surfaces in Principal Stress Space 


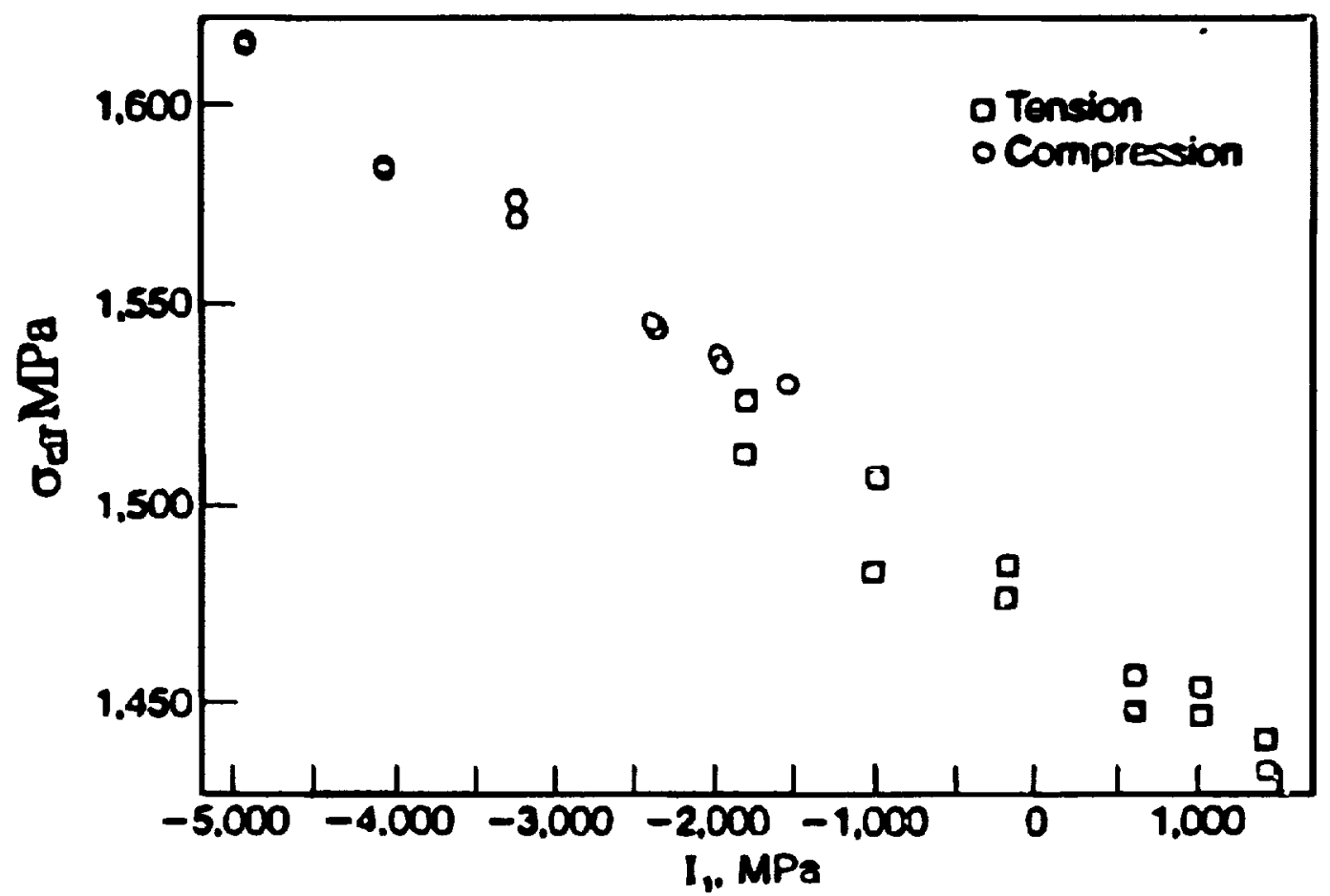

Figure 2 Dependence of Yielding on Mean Stress in 4330 Steel [10] 


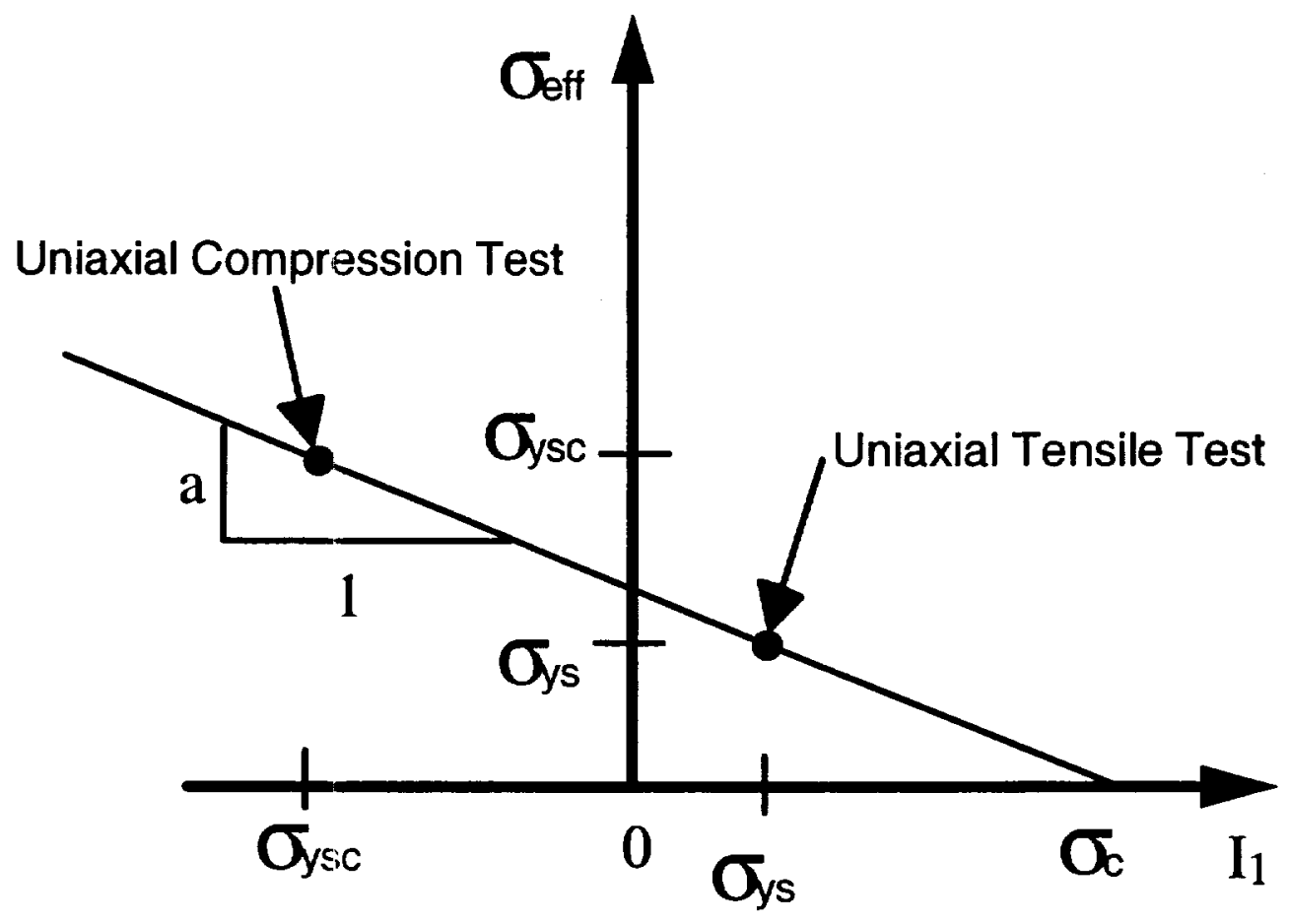

Figure 3 Schematic of $\sigma_{\text {rff }}$ versus $\mathrm{I}_{1}$ 


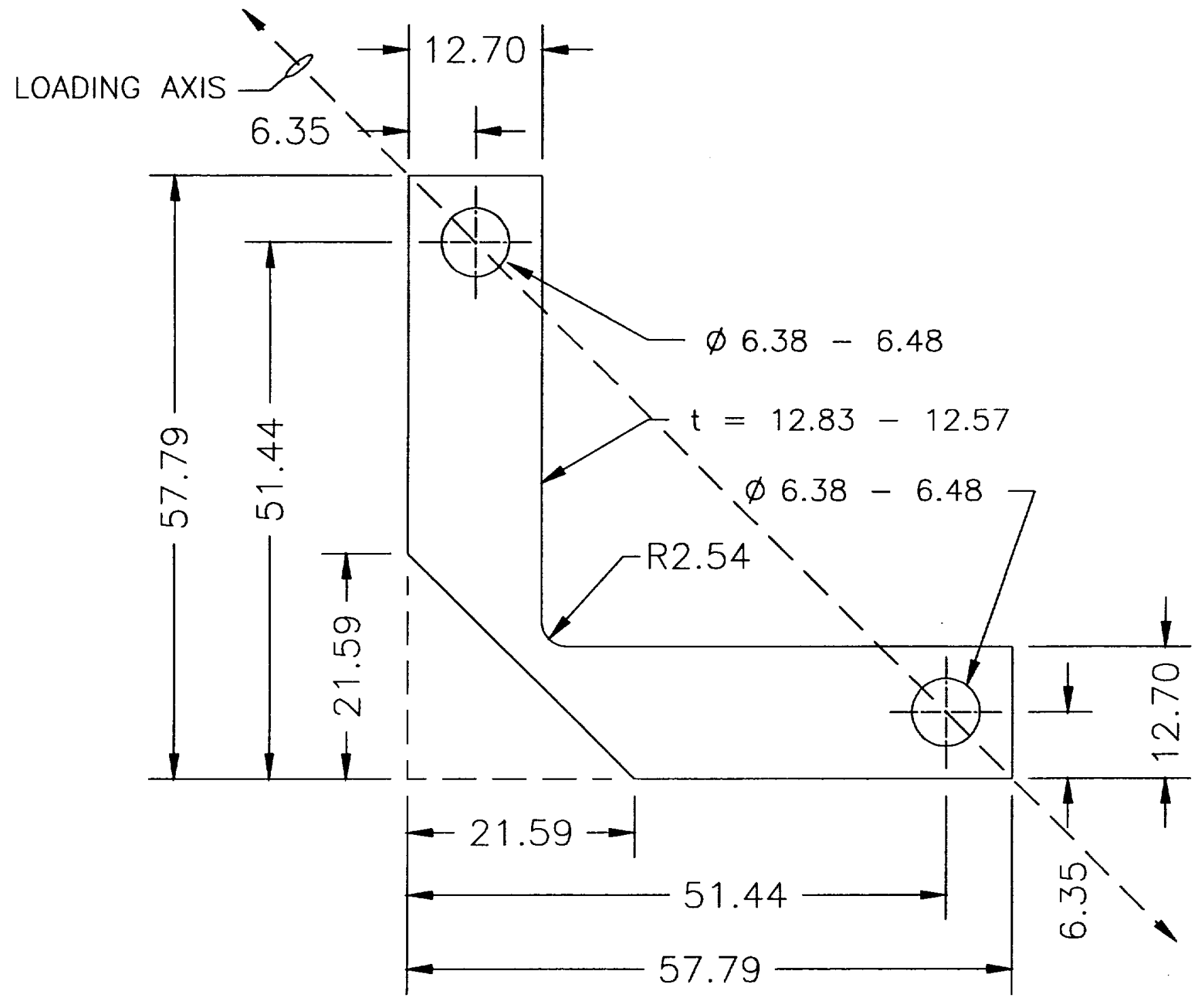

Figure 4 Engineering Drawing of the Equal Arm Bend Specimen (Dimensions in mm) 

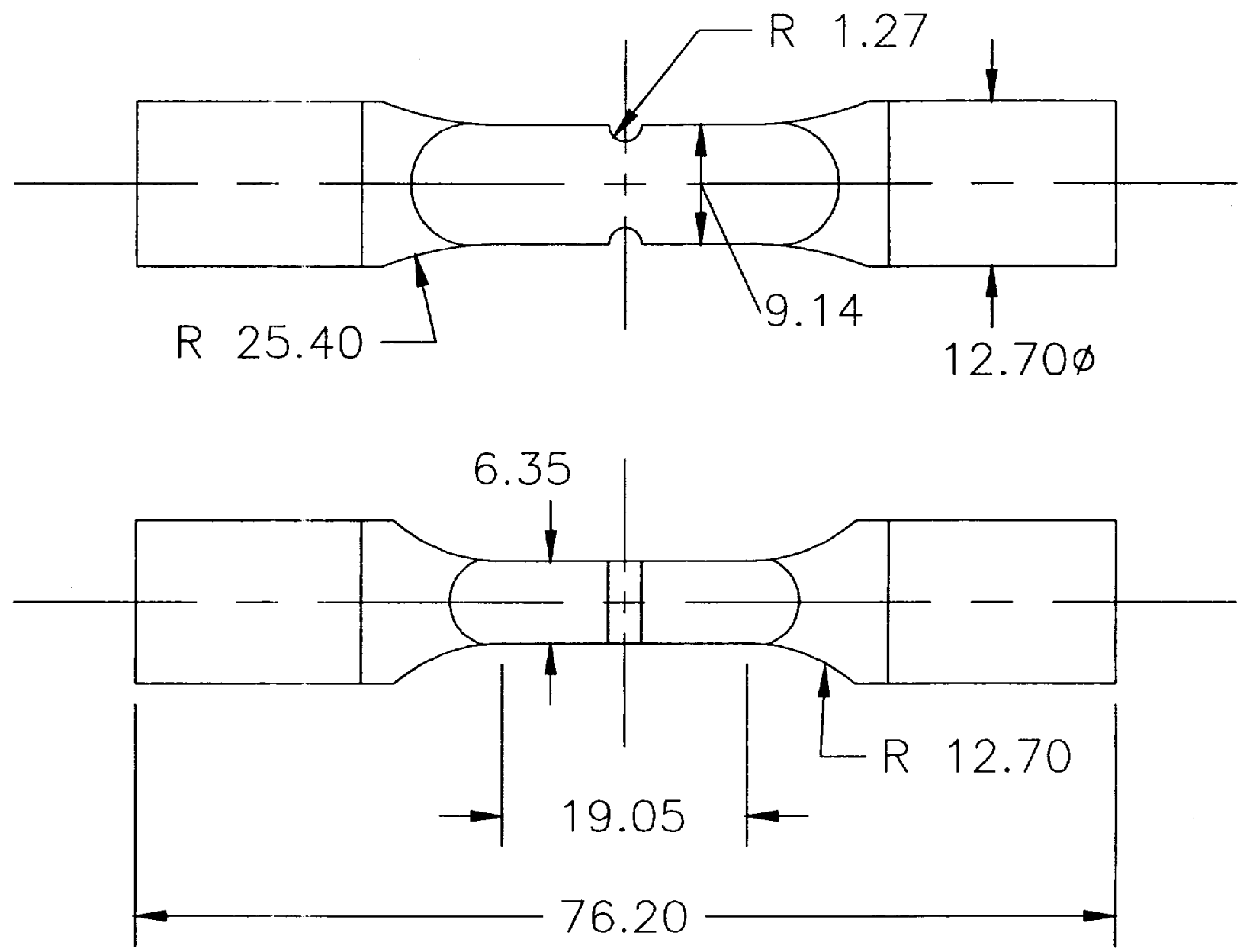

Figure 5 Engineering Drawing of the DENT Specimen (Dimensions in $\mathrm{mm}$ ) 


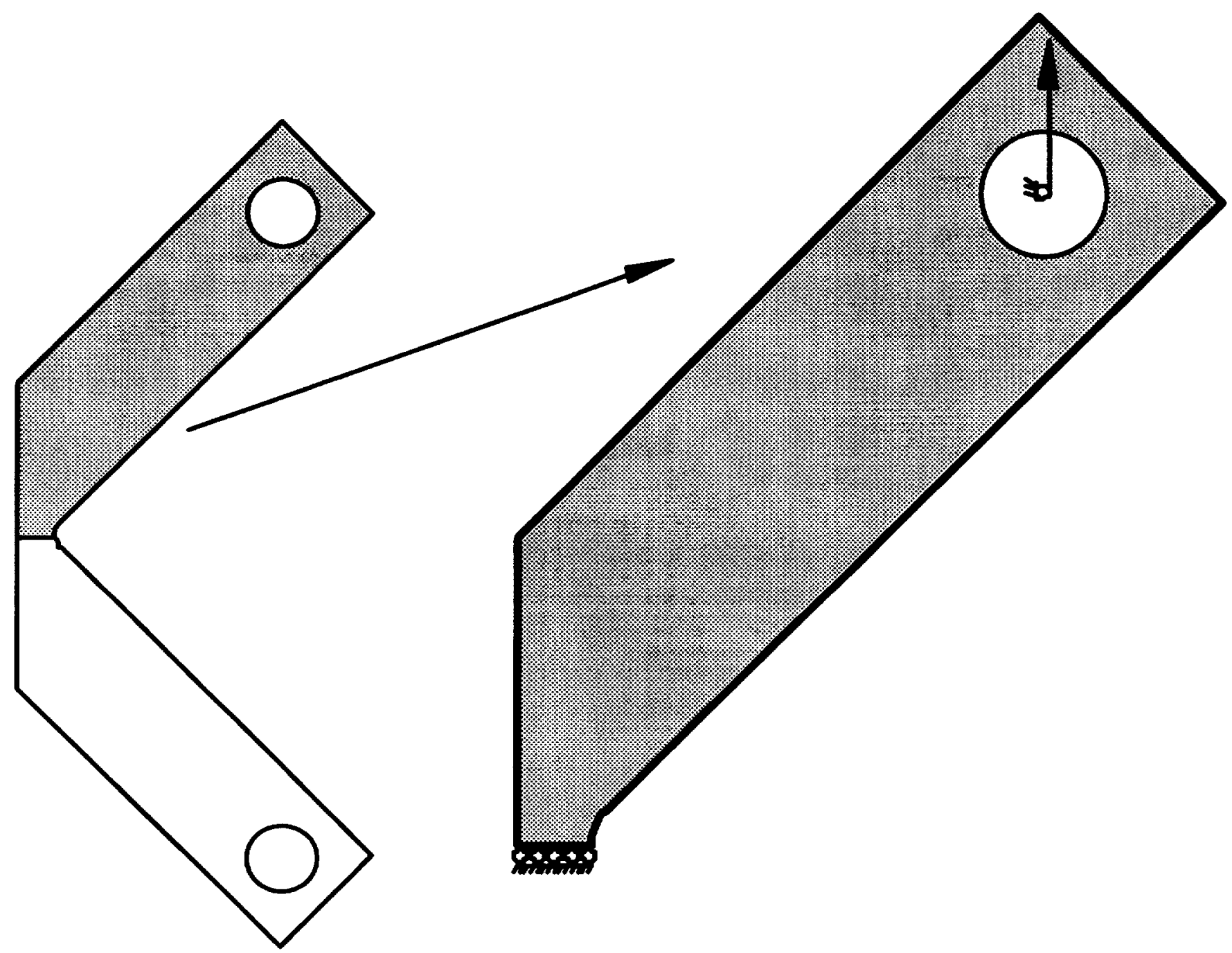

Figure 6 Schematic of the Equal-Arm Bend FEM Utilizing One Symmetry Plane 


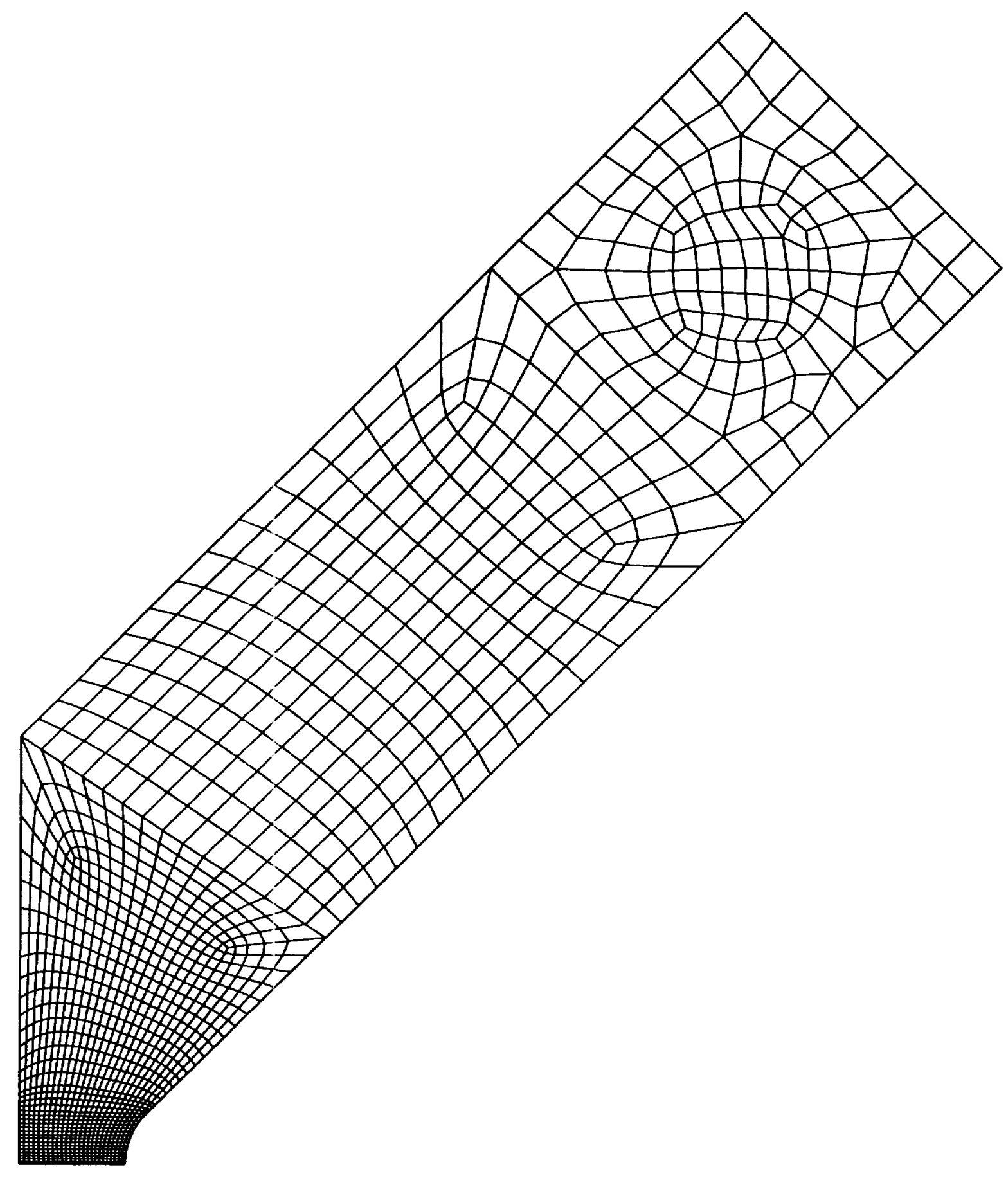

Figure 7 Equal Arm Bend Finite Element Model Utilizing One Plane of Symmetry 


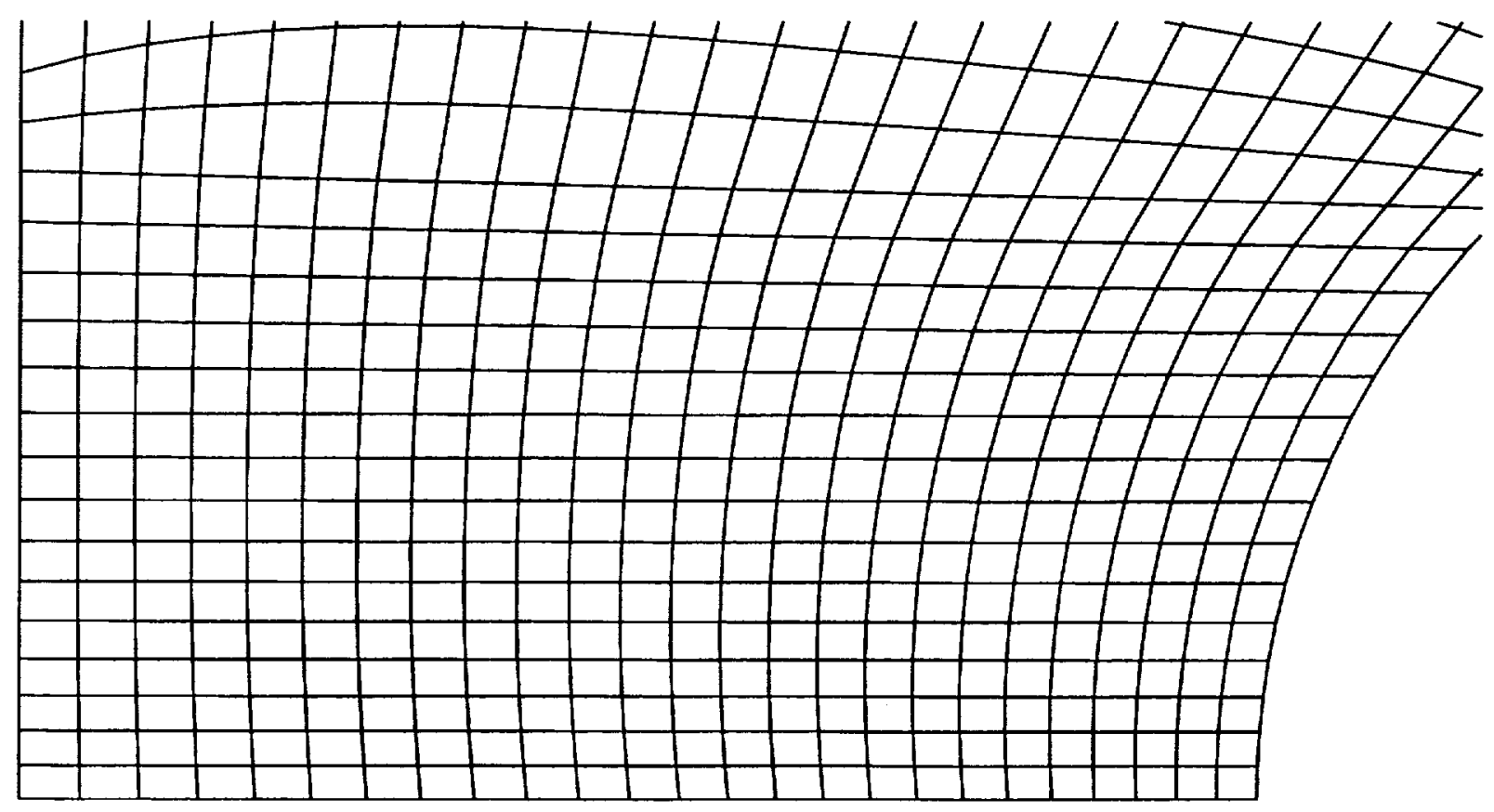

Figure 8 Mesh in the Fillet Region of the Equal Arm Bend Specimen 


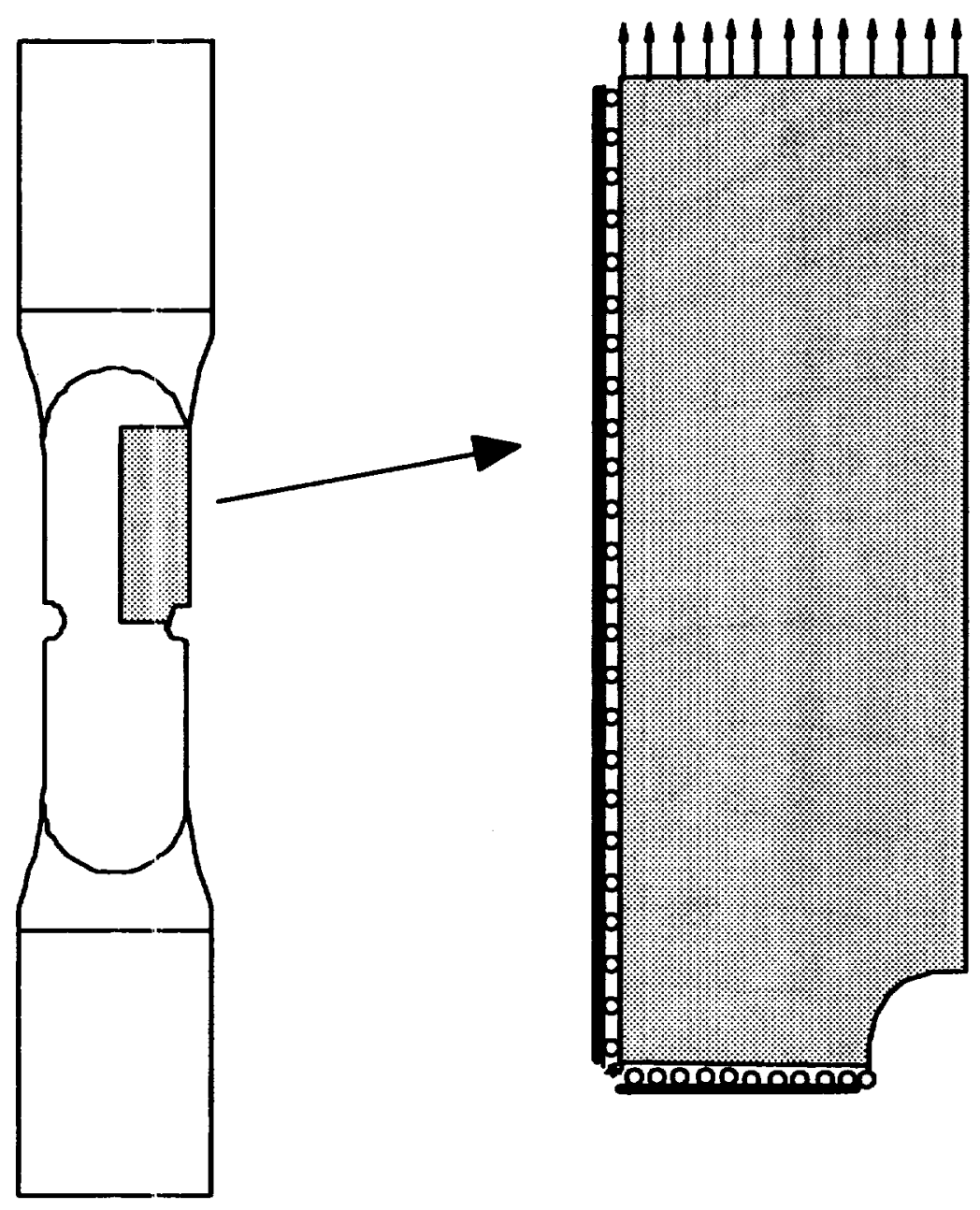

Figure 9 Schematic of the 2-D DENT FEM Utilizing Two Symmetry Planes 


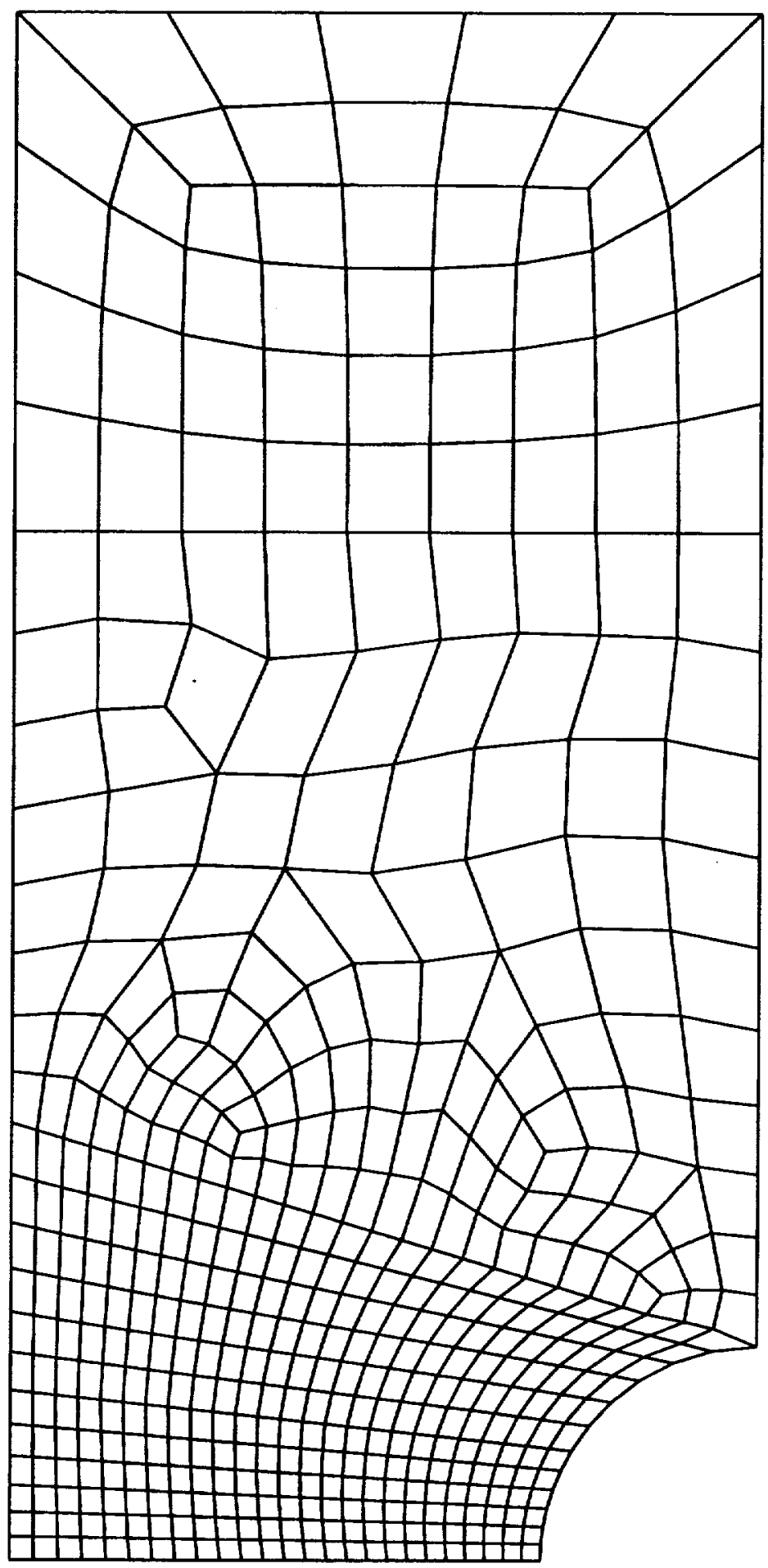

Figure 10 Illustration of the 2-D DENT FEM 


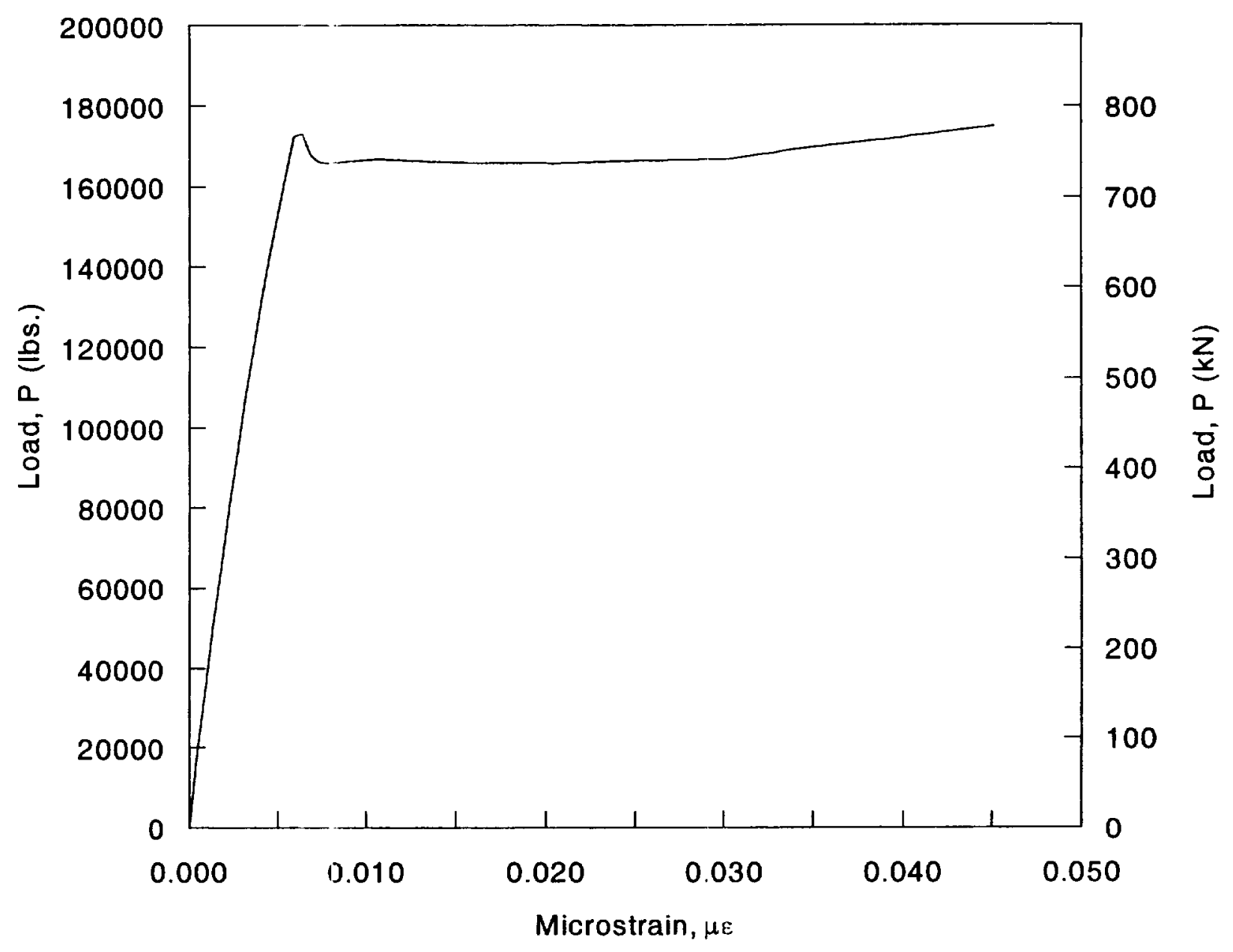

Figure 11 True Stress versus True Strain Curve for IN100 at Room Temperature [22] 


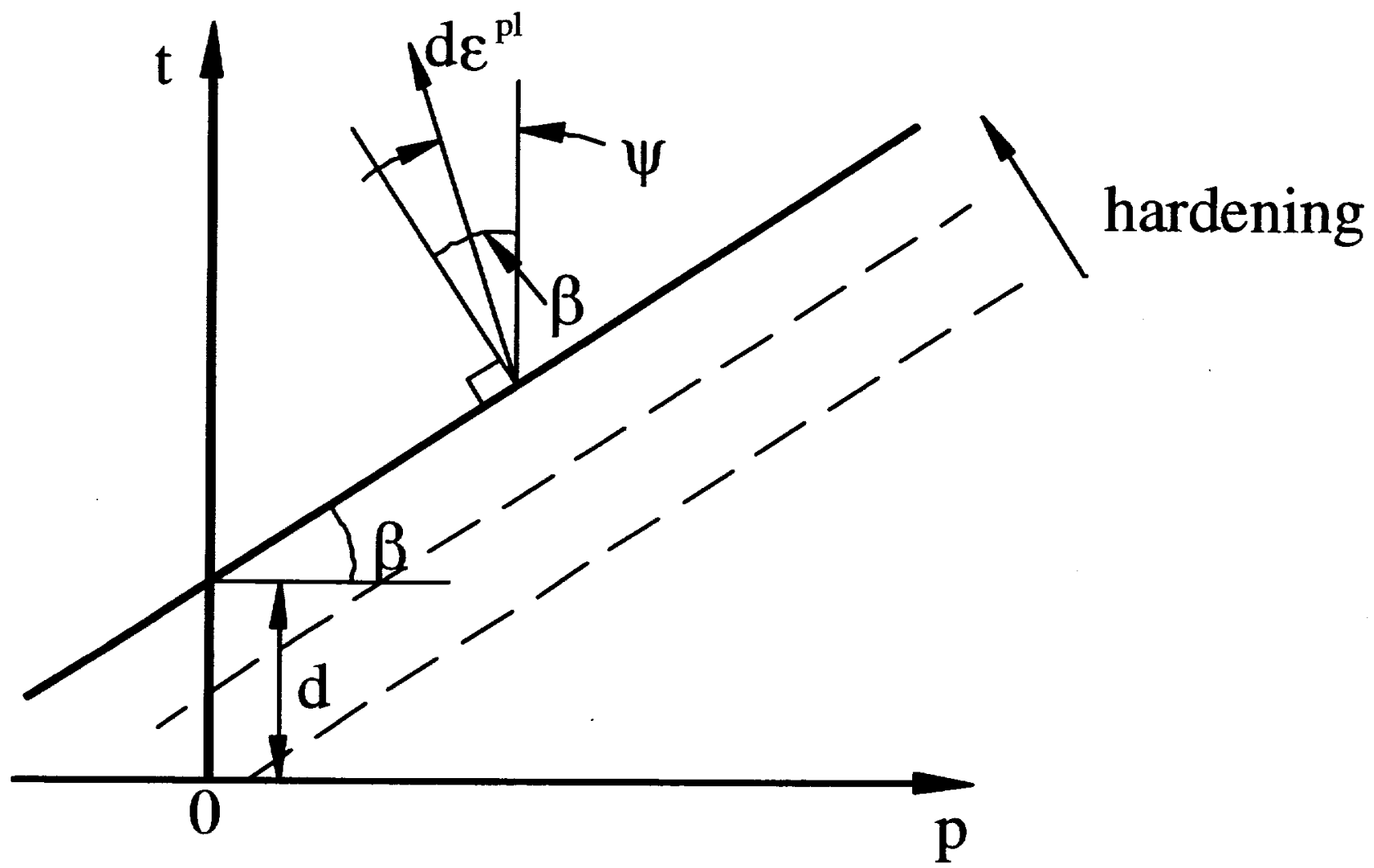

Figure 12 Linear Drucker-Prager Model: Yield Surface and Flow Direction in the $p$ - $t$ Plane (Adapted from [5]) 


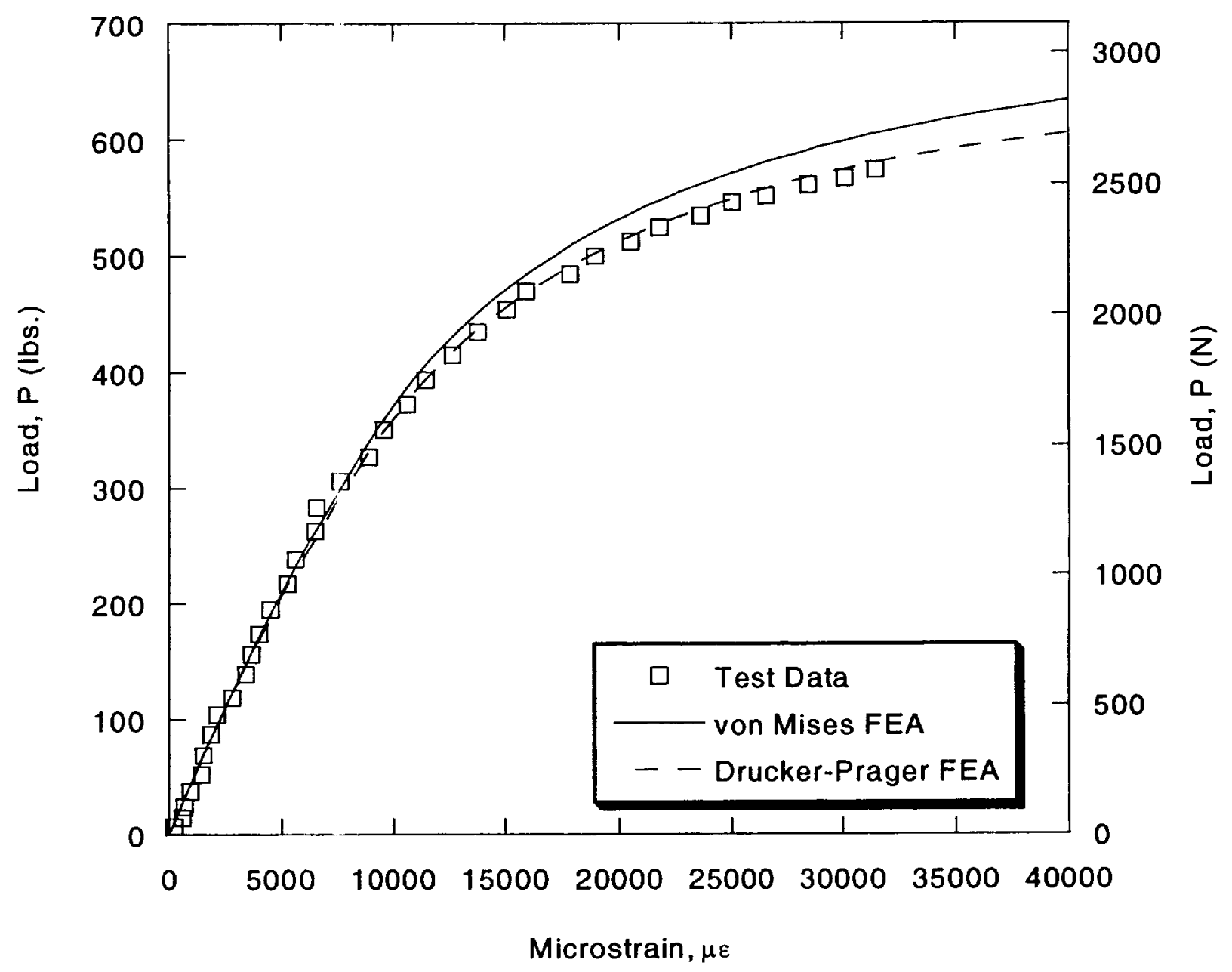

Figure 13 Load-Microstrain Results for the Equal-Arm Bend Specimen 


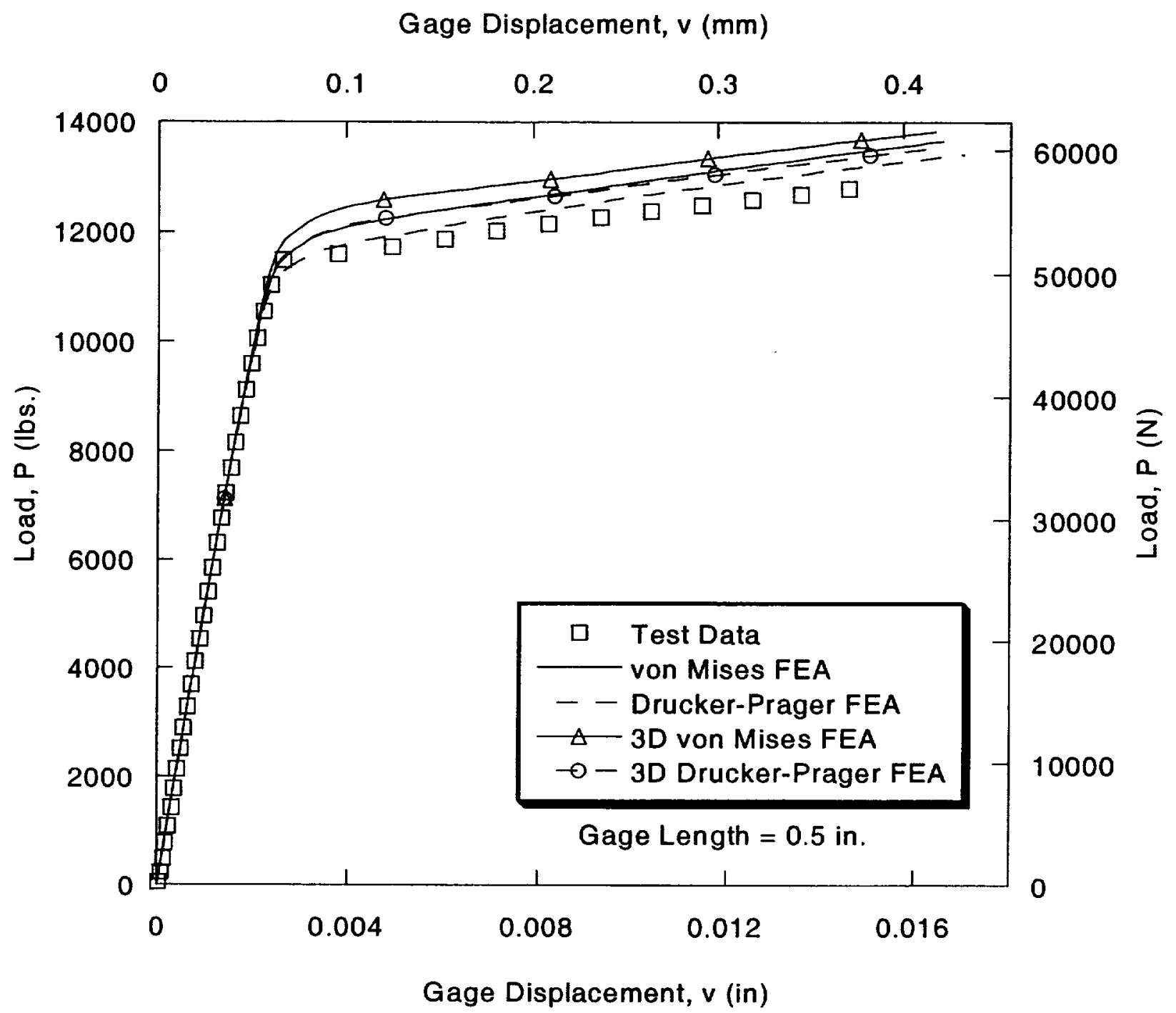

Figure 14 Load-Displacement Results for the DENT Specimen 